\title{
Multiparticle entanglement and its experimental detection
}

\author{
W. Dür and J. I. Cirac \\ Institut für Theoretische Physik, Universität Innsbruck, A-6020 Innsbruck, Austria
}

(October 21, 2018)

\begin{abstract}
We discuss several aspects of multiparticle mixed state entanglement and its experimental detection. First we consider entanglement between two particles which is robust against disposals of other particles. To completely detect these kinds of entanglement, full knowledge of the multiparticle density matrix (or of all reduced density matrixes) is required. Then we review the relation of the separability properties of $l-$ partite splittings of a state $\rho$ to its multipartite entanglement properties. We show that it suffices to determine the diagonal matrix elements of $\rho$ in a certain basis in order to detect multiparticle entanglement properties of $\rho$. We apply these observations to analyze two recent experiments, where multiparticle entangled states of 3 (4) particles were produced. Finally, we focus on bound entangled states (non-separable, non-distillable states) and show that they can be activated by joint actions of the parties. We also provide several examples which show the activation of bound entanglement with bound entanglement.
\end{abstract}

03.67.-a, 03.65.Bz, 03.65.Ca, 03.67.Hk

\section{INTRODUCTION}

Entanglement is at the heart of Quantum Information theory. In recent years, there has been an ongoing effort to characterize quantitatively and qualitatively entanglement. While for bipartite systems essential parts of this problem are solved, many questions remain still open for multipartite systems. In this case, there exist several possible approaches to identify different kinds of multiparticle entanglement (MPE), and many interesting phenomena related to MPE have been discovered [1 6 ].

In this work, we review some possible approaches to identify different kinds of MPE and discuss its experimental detection.

\section{A. Bipartite Entanglement, Separability and Distillability}

Let us start with the simplest case of bipartite systems and review some basic concepts related to bipartite entanglement. Let $A$ and $B$ be two spatially separated systems of dimension $d_{A}\left[d_{B}\right]$ respectively. A state $\rho$ is said to be separable if it can be written as a convex combination of product states, i.e.

$$
\rho=\sum_{i} p_{i}\left|a_{i}\right\rangle_{A}\left\langle a_{i}|\otimes| b_{i}\right\rangle_{B}\left\langle b_{i}\right| .
$$

In case this is not possible, $\rho$ is said to be entangled. Note that separable states $\rho$ are states which can be prepared locally by the parties, i.e. $\rho$ is only classically correlated. As inseparable (entangled) states are very interesting, both from a fundamental and from a practical point of view, one of the main problems in Quantum Information Theory is the problem of establishing whether a given state $\rho$ is separable or not. We have that condition (11) is in general very difficult to check, as there exist (in general) infinitely many ways to write a given density operator $\rho$ as a convex combination of (possible entangled) pure states. However, the problem of separability has been extensively studied in recent years [7], and in the case of two qubits $\left(d_{A}=d_{B}=2\right)$, necessary and sufficient conditions for separability have been obtained [8,9]. In particular, for two qubits one can use the partial transposition criterium [8,9] which states that (i) $\rho$ is separable iff $\rho^{T_{A}} \geq 0$ [9]. Here, $T_{A}$ denotes transposition in $A$ in a given orthonormal basis $S_{A}=\{|k\rangle\}_{k=1}^{d_{A}}$, and $X \geq 0$ means that all eigenvalues of $X$ are $\geq 0$. For higher dimensional systems $\left(d_{A}, d_{B}>2\right)$, positivity of the partial transposition is only a necessary, but not sufficient condition for separability.

For inseparable (entangled) density operators $\rho$, one may also ask whether the entanglement contained in $\rho$ can be distilled. That is, whether out of (arbitrary) many copies of $\rho$, a maximally entangled state (MES) such as the singlet state $\left|\Psi^{-}\right\rangle=(|01\rangle-|10\rangle) / \sqrt{2}$ shared by the parties $A$ and $B$ can be created by means of local operations and classical communication. In case this is possible, $\rho$ is said to be distillable. Again, for two qubits it turns out that the partial transposition provides a necessary and sufficient condition for distillability: (ii) $\rho$ is distillable iff $\rho^{T_{A}} \geq 0$ [10]. For higher dimensional systems $\left(d_{A}, d_{B}>2\right)$, non positive partial transposition is a necessary, but not sufficient condition for distillability.

The partial transposition criterium (i) and (ii) thus provides a necessary and sufficient condition for separability and distillability for two qubit systems.

\section{B. Multiparticle entanglement}

The aim of this paper is to extend these ideas to multiparticle systems, in particular to study separability and distillability properties of multiparticle systems. However, there are various aspects of multiparticle entanglement. For example, there exist obviously many different kinds of entanglement in a multiparticle system, as one may have bipartite entanglement shared by, say, parties 
$A_{1}$ and $A_{2}$ as well as bipartite entanglement shared by two other parties, say $A_{2}$ and $A_{3}$. In addition, there exist true $N$-partite entanglement, for example MES of $N$ particles such as the Greenberger-Horne-Zeilinger (GHZ) state 11

$$
|G H Z\rangle=\frac{1}{\sqrt{2}}\left(\left|0^{\otimes N}\right\rangle+\left|1^{\otimes N}\right\rangle\right) .
$$

Concerning for example the question of distillability, one may consider distillability of bipartite entanglement between pairs of particles or of true $N$-partite entanglement between a group of particles. In both cases, one may either ignore the remaining particles or allow them to assist the other parties in order to distill a MES. On may also consider partitions of the system, i.e. allowing some of the parties to act together and perform joint operations, and determine the distillability (and separability) properties with respect to this partitions, which in turn provide information about the entanglement properties of the whole system. Each of the situations just described is concerned with a different aspect of multiparticle entanglement, and will be discussed in more detail in the following.

From an experimental point of view, it is of particular interest to detect whether a $N$-particle state is distillable to a MES of $N$-particles. We will provide a simple, sufficient criteria which allows - without full knowledge of the density matrix - to detect true $N$-qubit entanglement. In addition, this criteria allows to detect different kinds of multiparticle entanglement as well. We also observe that there exist more kinds of multipartite entanglement then the obvious ones already mentioned previously (all possible combinations of maximally entangled $l$-partite states for different $l$ ). In particular, we consider bound entangled states, i.e. non separable, non distillable states and show that they can be activated under certain circumstances. We provide examples illustrating quite surprising effects related to bound entanglement and its activation.

This paper is organized as follows. We start in Sec. II by discussing bipartite aspects of MPE, that is entanglement which is robust against disposal of particles. We discuss the necessary information which is required to detect these aspects of MPE. In Sec. III, we choose a different approach and concentrate on $l$-partite aspects of MPE. Using $l$-partite splittings of the system, we show how to completely determine the separability and distillability properties of a certain family of states, i.e its MPE properties. Using these results, we provide a simple (sufficient) criteria to (experimentally) detect different kinds of MPE. We illustrate this method by applying it to two recent experiments, where MES of 3 (4) particles respectively were created. Finally, in Sec. IV we focus on an interesting phenomena related to MPE, namely on bound entanglement and its activation. In particular, we show that bound entanglement can sometimes be activated by joint actions of some of the parties or alternatively with help of a different kind of bound entanglement. We give several examples to illustrate these effects.

\section{ENTANGLEMENT WHICH IS ROBUST AGAINST DISPOSAL OF PARTICLES}

In this Section, we concentrate on bipartite aspects of multipartite entanglement, in particular on bipartite entanglement which is robust against disposal of particles. We consider $N$ spatially separated parties $A_{1}, \ldots, A_{N}$, each possessing a qubit.

We say that two particles are (bipartite) entangled if

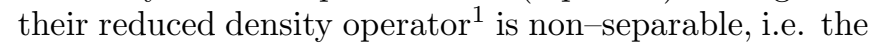
two particles share entanglement, independent what happens to the remaining particles. When considering the reduced density operator of two parties, we deal with the situation where the information about all remaining particles is not accessible (or the remaining parties are not willing to cooperate). Such a definition is very suitable from a practical point of view, as there are certain multipartite scenarios where one is interested in entanglement properties of pairs of parties, which are independent of other parties. In addition, in certain experiments one may be faced with such a situation, e.g. when one of the particles escapes from a trap. The remaining particles should then be described by the reduced density operator. Note that in this sense, the GHZ state (2) contains no (bipartite) entanglement at all, as all reduced density operators are separable. However, the GHZ state can be regarded as MES of $N$ particles in several other senses 12.

\section{A. Entanglement molecules}

In [6], it was shown that there exist $N$-particle states which are still entangled when tracing out any $(N-2)$ particles, i.e. there states where all particles are entangled with all other particles. In addition, it was shown there that there exist $N$-partite states $\rho$ where one can choose for each of the $N(N-1) / 2$ reduced density operators $\rho_{k l}$ independently whether it should be separable or inseparable. This allows to build general structures of $N$ particle states, which were called 'Entanglement molecules' in [6].

The following family of $N$ qubit states includes all possible configurations of 'Entanglement molecules' [6. First we specify for each of the reduced density operators $\rho_{k l}$ whether it should be distillable or not [13], i.e.

\footnotetext{
${ }^{1}$ Given a $N$-partite state $\rho$, the reduced density operator $\rho_{12}$ of party $A_{1}$ and $A_{2}$ is defined as $\rho_{12} \equiv \operatorname{tr}_{3, \ldots, N}(\rho)$. The operator $\rho_{12}$ is separable if it can be written as a convex combination of product states.
} 
whether entanglement between the parties $A_{k}$ and $A_{l}$ can be distilled — without help of the remaining parties or not. Let $I=\left\{k_{1} l_{1}, \ldots, k_{M} l_{M}\right\}$ be the set of all those pairs where distillation should be possible, i.e. for $k l \in I$, we have that $\rho_{k l}$ is distillable. We define the state

$$
\left|\Psi_{i j}\right\rangle \equiv\left|\Psi^{+}\right\rangle_{i j} \otimes|0 \ldots 0\rangle_{\text {rest }}
$$

that is the particles $A_{i}$ and $A_{j}$ are in a MES, namely $\left|\Psi^{+}\right\rangle=1 / \sqrt{2}(|01\rangle+|10\rangle)$, and the remaining particles are disentangled from each other and from $A_{i} A_{j}$. The family of states

$$
\rho_{I}=\frac{1}{M} \sum_{k l \in I} x_{k l}\left|\Psi_{k l}\right\rangle\left\langle\Psi_{k l}\right|,
$$

has the desired properties, which can be checked [6] by calculating the reduced density operators $\rho_{k l}$ and using the partial transposition criterium. We have that $M \equiv \sum_{k l \in I} x_{k l}$ is a normalization factor. The bipartite aspects of multipartite entanglement were also analyzed in 14.

\section{B. Experimental detection}

Given a $N$-qubit state $\rho$, how can we determine its (bipartite) entanglement properties ? One possibility is to completely determine the $N$-partite density matrix of $\rho$. Given $\rho$, one can easily calculate all possible reduced density operators $\rho_{k l}$ and determine the separability properties of each $\rho_{k l}$. Due to the fact that we deal with qubits, one can use the partial transposition criterium (see Sec. IA to determine for each of the reduced density operators $\rho_{k l}$ whether it is separable or distillable. In case $\rho_{k l}$ is inseparable, a MES shared by the parties $A_{k}$ and $A_{l}$ can be distilled.

However, it is rather difficult to completely determine the density matrix of $N$-qubit system, which is required in the procedure described above. Alternatively, one can concentrate from the very beginning on the properties of the reduced density operators $\rho_{k l}$, i.e. ignoring the remaining particles and just measuring the bipartite density operator $\rho_{k l}$. In this case, all $N(N-1) / 2$ different reduced density operators have to be determined independently and can then be analyzed using the partial transposition criterium.

Still, it might be too demanding to completely determine the density matrix of a two qubit system, which is necessary to completely determine the separability properties of this system. However, in order to detect entanglement in a two-qubit system, it suffices to show that the Fidelity $F$, i.e. the overlap with an arbitrary MES, fulfills $F>1 / 2$. Note however that this is a sufficient condition for inseparability (distillability), which is in general not necessary. So one can alternatively measure the overlap of each of the reduced density operators $\rho_{k l}$ with a MES. Observing that for a given $\rho_{k l}, F>1 / 2$ implies that out of $\rho_{k l}$ a MES shared among $A_{k}$ and $A_{l}$ can be distilled. However, when one finds $F \leq 1 / 2$, nothing can be concluded about the separability properties of $\rho_{k l}$.

Establishing the (bipartite) entanglement properties of a state $\rho$ is however not the only possibility to determine the multipartite entanglement (MPE) properties of $\rho$. The bipartite entanglement properties, i.e. the properties of the reduced density operators $\rho_{k l}$, are only a certain aspect of the MPE properties of $\rho$. There are other aspects of MPE and alternative ways to detect the presence of MPE, which will be discussed in the next Section.

\section{L-PARTITE ASPECTS OF MULTIPARTICLE ENTANGLEMENT}

In this section we first review the concepts of $l$-partite splittings, $l$-separability and distillability. These properties can be used to completely characterize the multiparticle entanglement properties of an arbitrary mixed state $\rho$ 15. We then review the properties of a family of $N$-qubit states $\rho_{N}$ introduced in Ref. [2] and completely determine the entanglement properties of this family. Finally we show that these results can be used to determine entanglement properties of general states $\rho$ without complete knowledge of the density matrix. In particular, it suffices to determine the diagonal matrix elements of $\rho$ in a certain basis in order to establish sufficient conditions for the presence of multipartite entanglement. We provide a simple receptive to experimentally detect different kinds of multipartite entanglement. We apply the results to two recently performed experiments 16,17 to illustrate the usefulness of our method.

\section{A. Bipartite and $l$-partite splittings}

Let us denote by $\mathcal{P}$ the set of all possible bipartite splittings of $N$ parties into two groups. For example, for 3 parties $\mathcal{P}$ contains the splittings $\left(A_{1} A_{3}\right)-\left(A_{2}\right),\left(A_{2} A_{3}\right)-$ $\left(A_{1}\right)$, and $\left(A_{3}\right)-\left(A_{1} A_{2}\right)$. We will denote these bipartite splittings by $P_{k}$, where $k=k_{1} k_{2} \ldots k_{N-1}$ is a chain of $N-1$ bits, such that $k_{n}=0,1$ if the $n$-th party belongs to the same group as the last party or not. For example, for 3 parties the splittings $\left(A_{1} A_{3}\right)-\left(A_{2}\right),\left(A_{2} A_{3}\right)-\left(A_{1}\right)$, and $\left(A_{3}\right)-\left(A_{1} A_{2}\right)$ will be denoted by $P_{01}, P_{10}$, and $P_{11}$, respectively. We will denote by $A$ the side of the splitting to which the party $N$ belongs and by $B$ the other side. In a similar way, one can consider $l$-partite splittings $S_{l}$, where the parties form exactly $l$ groups. In the following, when we consider $l$-partite splittings, the parties in each of the $l$ groups will be allowed to act together (i.e. to perform joint operations). 


\section{B. $l$-separability and distillability}

Here we review the notion of separability and distillability in the case of multiparticle systems. We consider $N$ parties, each holding a system with dimension $d_{i}$, i.e. $\mathcal{H}=C^{d_{1}} \otimes \ldots \otimes \mathbb{C}^{d_{N}}$. We call $\rho$ fully separable if it can be written as a convex combination of (unnormalized) product states, i.e.

$\rho=\sum_{i}\left|a_{i}\right\rangle_{\text {party } 1}\left\langle a_{i}|\otimes| b_{i}\right\rangle_{\text {party } 2}\left\langle b_{i}|\otimes \ldots \otimes| n_{i}\right\rangle_{\operatorname{party} N}\left\langle n_{i}\right|$.

In the following, we will consider a system of $N$ qubits, each hold by one of the parties $A_{1}, A_{2}, \ldots, A_{N}$. In this case, $d_{1}=d_{2}=\ldots d_{N}=2$. A state $\rho$ is called $k^{-}$ separable with respect to a specific $k$-partite splitting iff it is fully separable in the sense that we consider $\rho$ as a $k$-party system, i.e. as a state in $\mathcal{H}=\mathbb{C}^{d_{1}} \otimes \ldots \otimes \mathbb{C}^{d_{k}}$. In order to completely determine the separability properties of a state $\rho$, one should determine the separability properties of all possible $l$-partite splittings for all $l \leq N / 2$. Based on this information, one can establish an hierarchic classification of the entanglement properties of $\rho$ (see Ref. 15] for details). It turns out that the separability properties of the different $l$-partite splittings for different $l$ are not independent of each other, which strongly simplifies the classification and reduces the number of possible classes. In some cases we will deal with in the following, it even suffices to determine the biseparability properties of a state, i.e. to establish the separability properties of all possible bipartite splittings. This is due to the fact that the $l$-separability properties in this case are completely determined by the biseparability properties of $\rho$.

In a similar way, one can establish the distillability properties of a state $\rho$. Given a bipartite splitting $P_{k}$, a state $\rho$ is called distillable with respect to the splitting $P_{k}$, if - out of $N$ identical copies of $\rho$ - the two groups $A$ and $B$ (which correspond to the two groups of the splitting) can create by means of local operations and classical communication a MES such as $\left|\Phi^{+}\right\rangle=1 / \sqrt{2}(|00\rangle+|11\rangle)$, shared among $A$ and $B$. Recall that the term "local" in this case refers to local operation with respect to the groups $A$ and $B$, but may involve joint operation on the particles within one group. In the case of distillability, it is not necessary to consider $l$-partite splitting and the possible creation of $l$-party GHZ states, as the creation of pairwise entanglement between any two out of $l$ parties is a necessary and sufficient condition for the distillation of a $l$-partite GHZ state shared among those parties [15]. However, one may ask whether two subgroups - not containing all parties - are capable of distilling a MES with help of the remaining parties. For a certain family of states, we will give necessary and sufficient conditions when this is possible.

\section{Family of states $\rho_{N}$}

Let us consider $\rho_{N}$, the family of $N$-qubit states introduced in [2]. We have that $\rho \in \rho_{N}$ if it can be written as

$$
\begin{aligned}
\rho= & \sum_{\sigma= \pm} \lambda_{0}^{\sigma}\left|\Psi_{0}^{\sigma}\right\rangle\left\langle\Psi_{0}^{\sigma}\right| \\
& +\sum_{k \neq 0} \lambda_{k}\left(\left|\Psi_{k}^{+}\right\rangle\left\langle\Psi_{k}^{+}|+| \Psi_{k}^{-}\right\rangle\left\langle\Psi_{k}^{-}\right|\right),
\end{aligned}
$$

where

$$
\left|\Psi_{k}^{ \pm}\right\rangle \equiv \frac{1}{\sqrt{2}}\left(\left|k_{1} k_{2} \ldots k_{N-1} 0\right\rangle \pm\left|\bar{k}_{1} \bar{k}_{2} \ldots \bar{k}_{N-1} 1\right\rangle\right),
$$

are GHZ-like states with $k=k_{1} k_{2} \ldots k_{N-1}$ being a chain of $N-1$ bits, and $\bar{k}_{i}=0,1$ if $k_{i}=1,0$, respectively. We have that $\rho_{N}$ is parameterized by $2^{N-1}$ independent real numbers. The labeling is chosen such that $\Delta \equiv$ $\lambda_{0}^{+}-\lambda_{0}^{-} \geq 0$. As we will see below, both the separability and distillability properties of the states belonging to this family are completely determined by the coefficients

$$
s_{k} \equiv\left\{\begin{array}{l}
1 \text { if } \lambda_{k}<\Delta / 2 \\
0 \text { if } \lambda_{k} \geq \Delta / 2
\end{array}\right.
$$

Let us emphasize that the notation used for the states of this family parallels the one used to denote the partitions $P_{k}$, i.e. there is a one to one correspondence between $P_{k}$ and $s_{k}$. Note that there are no restrictions to the values of these coefficients; that is, for any choice of $\left\{s_{k}\right\}$ there always exists a state $\rho \in \rho_{N}$ with these values. We will now summarize the properties of states belonging to the family (6) 15, A4:

(i) Depolarization: An arbitrary state $\rho$ can be depolarized to the standard form (6) by a sequence of $N$-local operations while keeping the values of $\lambda_{0}^{ \pm} \equiv$ $\left\langle\Psi_{0}^{ \pm}|\rho| \Psi_{0}^{ \pm}\right\rangle$and $2 \lambda_{j} \equiv\left\langle\Psi_{j}^{+}|\rho| \Psi_{j}^{+}\right\rangle+\left\langle\Psi_{j}^{-}|\rho| \Psi_{j}^{-}\right\rangle$unchanged [15].

(ii) Separability: For any bipartite splitting $P_{k} \in \mathcal{P}$, and $\rho \in \rho_{N}$ we have $\rho^{T_{A}} \geq 0 \Leftrightarrow s_{k}=0 \Leftrightarrow \rho$ is separable with respect to this splitting 2 15. More generally, $\rho \in \rho_{N}$ is $l$-separable with respect to a specific $l$-partite splitting $S_{l}$ iff all bipartite splittings $P_{k}$ which contain ${ }^{\beta}$ $S_{l}$ are separable (have $s_{k}=0$ ).

(iii) Distillability: Let $\rho \in \rho_{N}, C=\left\{A_{i_{1}}, \ldots, A_{i_{M}}\right\}$ and $D=\left\{A_{j_{1}}, \ldots, A_{j_{L}}\right\}$ be two disjoint groups of $M$ and

\footnotetext{
${ }^{2} \rho^{T_{A}}$ denotes the partial transposition with respect to the parties $A$. For the definition of partial transposition in multiparticle systems see 8,15. The relation between subsystem $A$ and $P_{k}$ is given in Sec. IIIA.

${ }^{3}$ A $l$-partite splitting $S_{l}$ is contained in a $k$-partite splitting $P_{k}$ iff $P_{k}$ can be obtained from $S_{l}$ by joining some of the parties of $S_{l}$.
} 
$L$ parties respectively, whereas the rest of the parties are separated. A MES between $C$ and $D$ can be distilled iff $\rho$ is non-separable with respect to all those bipartite splittings $P_{k}$ in which the groups $C$ and $D$ are located on different sides (i.e. all corresponding $s_{k}=1$ ). It follows that $\rho$ is distillable with respect to a bipartite splitting $P_{k} \Leftrightarrow s_{k}=1$ [4].

Note that (ii) and (iii) completely determine the separability and distillability properties of an arbitrary state $\rho \in \rho_{N}$ and thus the multipartite entanglement properties of this state. We also have that (iii) already implies complete knowledge about the distillability of $k$-partite GHZ states, as the creation of pairwise entanglement between any two out of $k$ parties is a necessary and sufficient condition for the distillation of a $k$-partite $\mathrm{GHZ}$ state shared among those parties [15].

\section{Implications for experimental detection of multipartite entanglement}

We have that (i-iii) together provide a simple criterium for the detection of multipartite entanglement for arbitrary mixed states $\rho$ : From (i) follows that any state $\rho$ is at least as entangled as the depolarized version $\tilde{\rho} \in \rho_{N}$ of $\rho$. This is due to the fact that a sequence of local operations may destroy some entanglement, but cannot create any new kind of entanglement which was not present in the initial state. This already gives us a receptive to detect different kinds of multipartite entanglement of an arbitrary state $\rho$ :

- Determine the following diagonal matrix elements of $\rho$ :

$$
\begin{aligned}
\lambda_{0}^{ \pm} & \equiv\left\langle\Psi_{0}^{ \pm}|\rho| \Psi_{0}^{ \pm}\right\rangle \\
2 \lambda_{j} & \equiv\left\langle\Psi_{j}^{+}|\rho| \Psi_{j}^{+}\right\rangle+\left\langle\Psi_{j}^{-}|\rho| \Psi_{j}^{-}\right\rangle \\
& =\langle j 0|\rho| j 0\rangle+\langle\bar{j} 1|\rho| \bar{j} 1\rangle .
\end{aligned}
$$

Note that determining $\lambda_{0}^{ \pm}$requires a measurement in an entangled basis (GHZ basis), while determining $\lambda_{j}$ corresponds to a measurement in a product basis. Recall that $|j 0\rangle=\left|j_{1} j_{2} \ldots j_{N-1} 0\right\rangle$ and $|\bar{j} 0\rangle=\left|\bar{j}_{1} \bar{j}_{2} \ldots \bar{j}_{N-1} 1\right\rangle$ (see (8)). Equivalently, it suffices to determine all diagonal matrix elements of $\rho$ in the standard basis plus one off-diagonal element, namely $|0 \ldots 0\rangle\langle 1 \ldots 1|$.

- Calculate $\Delta=\lambda_{0}^{+}-\lambda_{0}^{-}=2 \operatorname{Re}(\langle 0 \ldots 0|\rho| 1 \ldots 1\rangle)$ and determine the coefficients $s_{k}$ given in (8). If at least one $s_{k}=1$, we have that $\rho$ is entangled.

- Use (i-iii) to determine the (minimal) entanglement properties of the state $\rho$. Note that obtaining $s_{k}=0$ for a certain bipartite splitting $P_{k}$ does not imply that $\rho$ is separable with respect to this splitting. It might well be that $\rho$ is inseparable (entangled) with respect to $P_{k}$, but the corresponding depolarized state $\tilde{\rho}$ is separable. However, obtaining $s_{k}=1$ ensures that a certain kind of entanglement is present in the state $\rho$ - namely that $\rho$ is inseparable with respect to the bipartite splitting $P_{k}$. In particular, one can distill a GHZ state from $\rho$ iff $s_{k}=1 \forall k$.

\section{E. Application to recent experiments}

Let us apply this method to two recent experiment performed by Sacket et al. [16] and Rauschenbeutel et al. [17.

In [16], the creation of an (mixed) entangled state $\rho$ of 4 ions, whose overlap with the GHZ state $\left|\Psi_{0}^{+}\right\rangle$is $F=0.57 \pm 0.02$, was reported. It was argued that it is sufficient to obtain $F>1 / 2$ in order to be sure that the state is 4 -partite entangled. This sufficient criteria is however - in some cases - much too demanding and can be relaxed using the results presented in this work. Imagine for example that the 4 qubit state $\rho$ is of the form

$$
\rho(x)=x\left|\Psi_{0}^{+}\right\rangle\left\langle\Psi_{0}^{+}\right|+\frac{1-x}{16} \mathbb{1}_{4}
$$

This is clearly a special case of the state $\rho_{4}$ with $\lambda_{0}^{-}=$ $\lambda_{j}=\frac{1-x}{16}, \lambda_{0}^{+}=x+\frac{1-x}{16}$ and thus $\Delta=x$. Using (ii) and (iii), we can state that $\rho(x)$ is fully non-separable and distillable to a 4 party GHZ state state iff $x>1 / 9$, which corresponds to $F>1 / 6$ [15]. Note that the bound $F>$ $1 / 2$ - which is independent of the number of parties $N$ corresponds to a worst case scenario, where it is assumed that $\lambda_{0}^{+}=F$ and the remaining weight is distributed on $\lambda_{0}^{-}$and one specific $\lambda_{k}$. In this case, we have for $F>1 / 2$ that $\Delta>2 \lambda_{k} \forall k$. If the remaining weight $(1-F)$ is however distributed on $\lambda_{0}^{-}$and more than one $\lambda_{k}$, it automatically follows that $\Delta>2 \lambda_{k} \forall k$ is already fulfilled for all $\lambda_{0}^{+} \equiv F>F_{0}$, where $F_{0}<1 / 2$. The weakest bound on the Fidelity $F$ can be obtained by assuming that the state $\tilde{\rho}_{N}$ is of the following form: $\lambda_{0}^{+}=F, \lambda_{0}^{-}=$ 0 and $2 \lambda_{k}=(1-F) /\left(2^{N}-2\right)$. This ensures that $\tilde{\rho}_{N}$ has $\Delta>2 \lambda_{k} \forall k$ and is thus distillable to a $N$-party GHZ state iff $F>1 /\left(2^{N}-1\right)$. For $N=4$, we obtain $F>1 / 15$. We thus have that additional knowledge of the shape of the state may relax the necessary conditions to ensure that a state is entangled.

Let us now focus on the specific experiment [16] and apply these observations. Unfortunately, the published experimental data is not sufficient to determine all coefficients $\lambda_{k}$. However, one can easily determine

$$
\begin{aligned}
\lambda_{0}^{ \pm}= & 1 / 2(\langle 0000|\rho| 0000\rangle+\langle 1111|\rho| 1111\rangle) \\
& \pm \operatorname{Re}(\langle 0000|\rho| 1111\rangle)=0.35 \pm 0.215( \pm 0.02),
\end{aligned}
$$

from which follows that $\Delta=0.43( \pm 0.02)$. In addition, one can also bound the other coefficients $\lambda_{k}$ and finds 18 


$$
\begin{aligned}
& 0 \leq 2 \lambda_{k} \leq 0.2( \pm 0.04) \text { iff } k \in\{001,010,100,111\} \\
& 0 \leq 2 \lambda_{k} \leq 0.1( \pm 0.02) \text { iff } k \in\{011,101,110\} .
\end{aligned}
$$

We thus have that $\Delta>2 \lambda_{k} \forall k$ as expected. Note however that a Fidelity $F<1 / 2$ would have been sufficient to ensure that the produced state is truly 4-partite entangled. Assume for example that white noise is added to the experimentally produced state, i.e. $\tilde{\rho}=x \rho+(1-x) / 16 \mathbb{1}_{4}$. Using the bounds on $\lambda_{k}$ just derived, we find that $\tilde{\rho}$ remains truly 4 -partite entangled for $x>0.58559$, which corresponds to a Fidelity of $F>0.3597$, significantly below $1 / 2$.

Our method should thus simplify the task to detect an entangled state of a larger number of particles $(N>4)$, as it relaxes the necessary conditions for the detection of true $N$-partite entanglement. Note that it would be highly desirable to measure all diagonal coefficients in the standard basis independently rather than Projections into subspaces $P_{j}$ with $j$ particles in $|0\rangle$ and $N-j$ particles in $|1\rangle$ as done in [16]. Doing so, one could determine the coefficients $\lambda_{k}$ directly and does not have to use a "worst case scenario" in order to establish bounds on $\lambda_{k}$ as we did here (see [18]). In addition, different kinds of entanglement which do not correspond to $N$-party GHZ entanglement can be detected as well. In the next section, we show that states showing these different kinds of entanglement may also be interesting to produce, as they provide examples for surprising effects such as the activation of bound entanglement.

One may also adopt this method to other experiments, such as the one performed by Rauschenbeutel et al. [17], where a maximally entangled state of three spin $\frac{1}{2}$ systems (two atoms plus one cavity mode) was created. Let us first adopt the notation used in $[17$ to the one used throughout this paper: $\left|{ }_{j}\right\rangle=|1\rangle,\left|-_{j}\right\rangle=-|0\rangle$, where e.g. $\left|+_{1}\right\rangle=\left|e_{1}\right\rangle,\left|+_{2}\right\rangle=\left(\left|g_{2}\right\rangle+\left|i_{2}\right\rangle\right) / \sqrt{2}$ and $\left|+_{C}\right\rangle=\left|g_{3}\right\rangle$ (see Equ. (3) and below in 17]). It follows that the longitudinal correlations given in Fig. 3 of 17] correspond to the diagonal matrix elements of $\rho$ in the basis (from left to right) $\{|011\rangle,|010\rangle,|001\rangle,|000\rangle,|111\rangle,|110\rangle,|101\rangle,|100\rangle\}$. From this we can determine

$$
\begin{aligned}
& 2 \lambda_{01}=0.14( \pm 0.04) \\
& 2 \lambda_{10}=0.155( \pm 0.04) \\
& 2 \lambda_{01}=0.128( \pm 0.04)
\end{aligned}
$$

From the transverse correlations we find

$$
\Delta=2 \operatorname{Re}\left(\langle 000|\rho| 111\rangle=2 V_{\perp}=0.28( \pm 0.04) .\right.
$$

Thus we have that $\Delta>2 \lambda_{k} \forall k$ and we can conclude that the experimentally detected state $\rho$ is in fact distillable to a 3-party GHZ state. Note that in [17], it was necessary to take known detection errors into account in order to obtain $F>0.5$. Here we can state that even without taking these errors into account, the state $\rho$ is true tripartite entangled, although its fidelity $F=0.43<1 / 2$.

\section{BOUND ENTANGLEMENT AND ITS ACTIVATION}

Let us now consider $N$ spatially separated parties, $A_{1}, \ldots, A_{N}$, who share $M$ identical copies of a $N$-qubit state $\rho$, where $M$ can be as large as we wish. This ensures that the parties can use distillation protocols [19] in order to obtain MES between some of them. In case this is possible, we say that the state $\rho$ is distillable (with respect to the specific parties which obtain the MES). If no MES shared between any two of the parties can be distilled and in addition the state $\rho$ is not fully separable (i.e. entangled), we say that $\rho$ is bound entangled (BE).

\section{A. Activating bound entanglement by joint actions}

Given a bound entangled state (BES), in some cases it is possible to activate the bound entanglement. We say that a BES can be activated if it becomes distillable once some of the parties join and form groups to act together. Note that instead of allowing some parties to join we could have allowed them to share some extra MES. In that case we would have the same situation given the fact that separated parties sharing MES can perform any arbitrary joint operation by simply teleporting 20] back and forth the states of their particles.

The first example of this kind was given in [2]. There it was shown that given a certain BES shared by 3 parties, providing some extra bipartite entanglement between $A_{1}$ and $A_{2}$ enables the 3 parties to create a tripartite $G H Z$ state.

In [3], Smolin presented another example of this kind involving 4 parties. This example has the additional feature that only a single copy of a BES $\rho$ is required in order to distill a MES shared by two of the parties (say $A_{1}$ and $A_{2}$ ) once the other two parties (say $A_{3}$ and $A_{4}$ ) are allowed to act together and perform joint operations.

Using states of the form (6), several examples showing the activation of different kinds of $\mathrm{BE}$ by joint actions of some of the parties were provided in [4. In addition, a systematic way for the construction of different kinds of activable BES was provided there. Let us review some of the examples given in [4]:

Example I: The state $\rho_{I}$ becomes distillable iff the parties form two groups with exactly $j$ and $N-j$ members, respectively. Furthermore, it does not matter which of the parties join in each group, but only the number of members. For example, if $N=8$ and $j=3$, we have that $\rho_{I}$ is distillable if exactly 3 and 5 parties join, but remains undistillable when the parties form two groups with 1-7, 2-6, 4-4 members, or if they form more than two groups. In particular, $\rho_{I}$ is not distillable if the parties remain separated from each other, which corresponds to having 8 groups. We can take as state $\rho_{I}$ one from the family $\rho_{N}$ which has $s_{k}=1$ iff the number of ones in $k$ is $j$ or $(N-j)$ and $s_{k}=0$ otherwise (this means that all 
bipartite splittings which contain exactly $j$ members in one group are distillable, and all others are separable).

Example II: The state $\rho_{I I}$ becomes distillable iff the parties form two groups, where the first group contains a specific set of $L$ parties $A=\left\{A_{k_{1}}, \ldots A_{k_{L}}\right\}$, and the second group contains the remaining parties. For all other configurations in groups $\rho_{I I}$ remains undistillable. For example, we have for $N=5$ and $A=\left\{A_{1}, A_{3}, A_{5}\right\}$ that $\rho_{I I}$ is distillable iff the the parties form two groups, $\left(A_{1} A_{3} A_{5}\right)-\left(A_{2} A_{4}\right)$, and not distillable otherwise. We can take $\rho_{I I} \in \rho_{N}$ such that $s_{k}=1$ only for one specific $P_{k}$. For $N=5$, choosing $s_{0101}=1$ ensures that $\rho_{I I}$ is inseparable and thus distillable with respect to the bipartite splitting $\left(A_{1} A_{3} A_{5}\right)-\left(A_{2} A_{4}\right)$ and separable (and thus undistillable) otherwise.

Example III: $\rho_{I I I}$ is a BES of $N=4$ parties for which once the parties $\left(A_{3} A_{4}\right)$ form a group, a GHZ-like state can be distilled among $A_{1}, A_{2}$, and the group $\left(A_{3} A_{4}\right)$, whereas it is undistillable whenever any other parties but $\left(A_{3} A_{4}\right)$ are joint. We choose $\rho_{I I I} \in \rho_{4}$ such that it is inseparable with respect to the bipartite splittings $\left(A_{1} A_{2}\right)$ $\left(A_{3} A_{4}\right),\left(A_{1}\right)-\left(A_{2} A_{3} A_{4}\right)$ and $\left(A_{2}\right)-\left(A_{1} A_{3} A_{4}\right)$ and separable with respect to all other bipartite splittings.

The described activation effects can be understood using (ii) and (iii) of Sec. III , together with the fact that when joining some of the parties, one may change the separability properties of certain bipartite splittings $P_{k}$ from separable to inseparable (see [4] for details).

We conclude that the experimental creation of nonmaximally entangled $N$-partite states (not all $s_{k}=1$ ) might be of interest as well, as those states can have quite surprising properties. Note however that in this case, it is essential that the produced states are of the form (6), which can be accomplished by physically implementing the depolarization procedure described in 15.

\section{B. Activating bound entanglement with bound entanglement}

Let us now consider the situation where the $N$ parties possess different kinds of BES, $\rho_{1}, \rho_{2}, \ldots \rho_{L}$, but this time remain spatially separated from each other. The parties again possess several copies of each of the states, i.e. $\rho_{i}^{\otimes M_{i}}$. By definition, it is clear that $\rho_{i}^{\otimes M_{i}}$ is not distillable for all $i$, i.e. the parties cannot create a MES if they have access to only one kind of BES.

However if the parties have access to all different kinds of BES, i.e they share the state

$$
\rho^{\prime}=\otimes_{i=1}^{L} \rho_{i}^{\otimes M_{i}},
$$

we will give examples were they can distill a MES between some of the parties or even a GHZ state shared among all the parties. This effect, namely that the tensor product of two BES is no longer necessarily a BES was discovered by Shor et al. [5] and was termed "Superactivation". We shall refer to this as activation of bound entanglement with bound entanglement.
Let us investigate the simplest example of a tripartite system, $N=3$. We consider a state $\rho_{1}$ which is inseparable with respect to the bipartite splitting $A-B C$ and separable with respect to the splittings $B-A C$ and $C-A B$. As shown in [4], such a state is BE (a necessary condition for distillation of a MES shared between any two of the three parties is that at least two of the bipartite splittings have to be inseparable). Now consider states $\rho_{2}$ and $\rho_{3}$ which are created from the state $\rho_{1}$ by cyclic permutations of the parties, i.e. $\rho_{2}\left[\rho_{3}\right]$ is inseparable with respect to the splitting $B-A C[C-A B]$ respectively. For a particular choice of the states $\rho_{1}, \rho_{2}, \rho_{3}$, the parties can create - once they have access to all three kinds of states - a state $\tilde{\rho}$ which is inseparable with respect to all three bipartite splittings and which is in addition distillable to a GHZ state. In fact, they just have to pick randomly one of the three states $\rho_{1}, \rho_{2}, \rho_{3}$ (this can be accomplished via classical communication only), i.e.

$$
\tilde{\rho}=\frac{1}{3}\left(\rho_{1}+\rho_{2}+\rho_{3}\right) .
$$

To be specific, choosing $\rho_{1}$ within the family of states (6),$N=3$ with the following coefficients

$$
\lambda_{0}^{+}=\frac{1}{3} ; \quad \lambda_{0}^{-}=\lambda_{2}=0 ; \quad \lambda_{1}=\lambda_{3}=\frac{1}{6}
$$

ensures (i) that $\rho_{1}$ and $\rho_{2}, \rho_{3}$ (created by cyclic permutations of the parties) are BE with separability properties with respect to the bipartite splittings as announced above. (ii) $\tilde{\rho}$ defined in (16) is again of the form (6) with coefficients

$$
\tilde{\lambda}_{0}^{+}=\frac{1}{3} ; \quad \tilde{\lambda}_{0}^{-}=0 ; \quad \tilde{\lambda}_{1}=\tilde{\lambda}_{2}=\tilde{\lambda}_{3}=\frac{1}{9},
$$

and is inseparable with respect to all bipartite splittings ( since $\Delta>2 \lambda_{k}$ ) and hence distillable to a GHZ state.

It is now straightforward to extend these ideas to more parties and to a more general setup. Therefore we consider a subfamily of $N$-qubit states of the form (6). We denote by $S$ all those bipartite splittings $P_{k}$ for which the state $\rho$ is inseparable (the corresponding $s_{k}=1$ ). For all other bipartite splittings $P_{k} \notin S, \rho$ is separable (the corresponding $s_{k}=0$ ). Let the number of separable bipartite splittings be $s>0$. We define $\Delta \equiv 1 /(s+1)$. The subfamily is defined by the following choice of parameters:

$$
\begin{gathered}
\lambda_{0}^{+}=\Delta ; \quad \lambda_{0}^{-}=0 ; \\
\lambda_{k}=0 \text { iff } P_{k} \in S \\
\lambda_{k}=\frac{\Delta}{2} \text { iff } P_{k} \notin S .
\end{gathered}
$$

In general, we can announce the following

Theorem 1: Given $L$ different kinds of BES $\rho_{1}, \ldots, \rho_{L}$ of the form (19), where $S_{j}$ denotes all bipartite splittings with respect to which $\rho_{j}$ is inseparable, one can create a state $\tilde{\rho}$ which is inseparable with respect to all those 
bipartite splittings where at least one of the states $\rho_{j}$ was inseparable, i.e. $\tilde{S}=\cup S_{j}$.

proof: We define

$$
\tilde{\rho}=\frac{1}{L} \sum_{j=1}^{L} \rho_{j},
$$

i.e. we pick randomly one of the states $\rho_{j}$ (which can be accomplished by classical communication) and show that $\tilde{\rho}$ has the desired properties. We have that $\tilde{\rho}$ is again of the form (6) and the coefficients $\tilde{\lambda}_{k}$ are given by the average of the coefficients $\lambda_{k, j}$ of the states $\rho_{j}$. We have to show that (i) $\tilde{\Delta}>2 \tilde{\lambda}_{k}$ iff $P_{k} \in \tilde{S} \equiv \cup S_{j}$ and (ii) $\tilde{\Delta} \leq 2 \tilde{\lambda}_{k}$ iff $P_{k} \notin \tilde{S}$. We have that $\tilde{\Delta}=1 / L \sum_{j=1}^{L} \Delta_{j}$. In case (i), we have that least one of the states $\rho_{j}$ is inseparable with respect to the splitting $P_{k}$. We assume without loss of generality that it is only one, namely $\rho_{1}$ and thus $\lambda_{k, 1}=0$ (the argument is exactly the same if more than one of the states $\rho_{j}$ are inseparable with respect to $P_{k}$ ). In this case we obtain for the corresponding $\tilde{\lambda}_{k}=1 / L \sum_{j=2}^{L} \Delta_{j} / 2$. Note that the sum runs from $j=2$ to $L$, which ensures that (i) is fulfilled - since $\left(\tilde{\Delta}-2 \tilde{\lambda}_{k}\right)=\Delta_{1} / L>0$. In case of (ii), i.e. $P_{k} \notin S_{j} \forall j$, we find $\tilde{\lambda}=1 / L \sum_{j=1}^{L} \Delta_{j} / 2$ and (ii) is fulfilled, which finishes the proof of our statement.

Given this theorem, it is now very easy to construct several examples which show the activation of bound entanglement with bound entanglement:

Example 1: We consider $N$ parties and assume that $N$ is even. We have $N / 2$ different BE states $\left\{\rho_{k}\right\}, k=$ $1,2, \ldots, N / 2$. If the parties have access to any $(N / 2-1)$ (or less) different kinds of BE states $\rho_{k}$, they cannot distill any entanglement. However, once the parties have access to all kinds of BE states $\rho_{k}$, they can create a state $\tilde{\rho}$ which is inseparable with respect to all bipartite splittings and thus distillable to a $N$-party GHZ state. The following choice of states has the announced properties: The state $\rho_{k}$ is of the form (19) and is inseparable with respect to all bipartite splittings which contain exactly $k$ parties on one side and $N-k$ parties on the other side and separable with respect to all other bipartite splittings. This ensures that all state $\rho_{k}$ are BE [4] and - according to Theorem 1 - the parties can create a state $\tilde{\rho}$ which is distillable to a GHZ state once they have access to all $N / 2$ different states $\rho_{k}$. If the access is limited to $(N / 2-1)$ or less different kinds of BE states $\rho_{k}$, one can easily check using (iii) of Sec. III that no entanglement can be distilled.

Example 2: In this example, we consider $N$ different BE states $\left\{\rho_{l}\right\}, l=1,2, \ldots, N$. Here, the state $\rho_{N}$ serves as a "key-state", as on one hand, access to $\rho_{N}$ together with access to the state $\rho_{l}$ enables the parties $A_{l}$ and $A_{N}$ to distill a MES. On the other hand, access to all states $\rho_{l}$ except $\rho_{N}$ does not allow the parties to distill any entanglement at all. If in addition also $\rho_{N}$ is accessible, a GHZ state shared by all the parties can be distilled (as party $A_{N}$ can create a MES shared with any party $\left.A_{l}\right)$. Such a situation can be established by the following choice of states: For $l \neq N$, the state $\rho_{l}$ is of the form (19) and is inseparable with respect to all bipartite splittings which have parties $A_{l}$ and $A_{N}$ on different sides, except the splittings $A_{l}$-rest and $A_{N}$-rest which as well as all the other splittings are separable. The state $\rho_{N}$ is also of the form (19) and is inseparable with respect to all splittings where exactly one particle is on one side and $N-1$ particles are on the other side. All states $\rho_{l}$ are BE, which can be checked using (ii) and (iii) of Sec. III Q. Applying Theorem 1, it is easy to observe the described activation effect.

Note that the activation of $\mathrm{BE}$ by joint actions may be combined with the activation of $\mathrm{BE}$ with $\mathrm{BE}$. This opens a huge variety of different examples, which can all be constructed using the results of Ref. [- 1 ] together with Theorem 1 and states of the form (19).

\section{SUMMARY}

We discussed several aspects of multipartite entanglement and its experimental detection. First we focused on bipartite aspects of MPE, which can be determined by investigating the bipartite reduced density operators of the multipartite systems. We then used $l$-partite splittings to establish the $l$-separability and distillability properties of a multipartite density operator $\rho$. For a certain family of states, we completely determined the separability and distillability properties using bipartite splittings only. Using this, we provided a simple method to determine whether a mixed state $\rho$ is multipartite entangled, and in addition to detect which kind of entanglement is present. We illustrated this method by revisiting two recent experiments. Finally, we focused on bound entangled states and the activation of $\mathrm{BE}$. We showed that $\mathrm{BE}$ can be activated by joint actions of the parties or with help of a different kind of BE itself.

\section{ACKNOWLEDGEMENTS}

We thank G. Vidal and J. Smolin for discussions. This work was supported by the Austrian Science Foundation under the SFB "control and measurement of coherent quantum systems (Project 11), the European Community under the TMR network ERB-FMRX-CT96-0087 and project EQUIP (contract IST-1999-11053), the European Science Foundation and the Institute for Quantum Information GmbH.

[1] A. Thapliyal, Phys. Rev. A 59 (1999) 3336; J. Kempe, Phys. Rev. A 60 (1999) 910; C. H. Bennett, D. P. DiVincenzo, T. Mor, P. W. Shor, J. A. Smolin, and B. M. 
Terhal, Phys. Rev. Lett. 83, 3081 (1999); D. P. DiVincenzo, T. Mor, P. W. Shor, J. A. Smolin, B. M. Terhal, quant-ph/9908070;

[2] W. Dür, J. I. Cirac, and R. Tarrach, Phys. Rev. Lett. 83, 3562 (1999).

[3] J. A. Smolin, quant-ph/0001001

[4] W. Dür and J. I. Cirac, Phys. Rev. A 62, 022302 (2000).

[5] P. W. Shor, J. A. Smolin and A. V. Thapliyal, quant$\mathrm{ph} / 0005117$.

[6] W. Dür, quant-ph/0006105

[7] for a review see M., P. and R. Horodecki, in "Quantum Information - basic concepts and experiments", in print (Springer, Berlin 2000); M. Lewenstein, D. Bruß, J. I. Cirac, B. Kraus, M. Kus, J. Samsonowicz, A. Sanpera and R. Tarrach, quant-ph/0006064

[8] A. Peres, Phys. Rev. Lett. 77, 1413 (1996).

[9] M. Horodecki, P. Horodecki and R. Horodecki, Phys. Lett. A223, 8 (1996).

[10] M. Horodecki, P. Horodecki and R. Horodecki, Phys. Rev. Lett. 78, 574 (1997).

[11] D. M. Greenberger, M. Horne, A. Zeilinger, Bell's theorem, Quantum Theory, and Conceptions of the Universe, ed. M. Kafatos, Kluwer, Dordrecht 69 (1989); D. Bouwmeester et al., Phys. Rev. Lett. 82 , 1345 (1999).

[12] N. Gisin and H. Bechmann-Pasquinucci, quant-ph 9804045

[13] Note that the inseparability of a certain reduced density operator $\rho_{k l}$ already implies that a MES — such as $\left|\Phi^{+}\right\rangle=1 / \sqrt{2}(|00\rangle+|11\rangle)$ - shared between parties $A_{k}$ and $A_{l}$ can be distilled (when allowing for several copies of the state), even without help of the remaining parties. This is due to the fact that for two qubit systems, inseparability is equivalent to distillability 10. In fact, the remaining parties can by no means prevent parties $A_{k}$ and $A_{l}$ to distill a MES.

[14] W. K. Wootters, quant-ph/0001114; M. Koashi, V. Buzek and N. Imoto, Phys. Rev. A 62, 050302(R) (2000); K. M. O'Connor and W. K. Wootters, quant-ph/0009041

[15] W. Dür and J. I. Cirac, Phys. Rev. A 61, 042314 (2000).

[16] C. A. Sackett et al., Nature 404, 256-259 (2000).

[17] A. Rauschenbeutel et al., Science 288, 2024-2028 (2000)

[18] In [16], only the probabilities $p_{l}$ to find $l$ ions in state $|0\rangle$ and $N-l$ ions in state $|1\rangle$ are given. For $l=1$, there are 4 states which might contribute to $p_{1}$, namely $\left|\phi_{i}^{1}\right\rangle \in\{|0111\rangle,|1011\rangle,|1101\rangle,|1110\rangle\}$. From the measured date we know that $p_{1}=P_{1} \rho P_{1}=$ $\operatorname{tr}\left(\sum d_{i j}^{1}\left|\phi_{i}^{1}\right\rangle\left\langle\phi_{j}^{1}\right|\right)$, where $P_{1}=\sum\left|\phi_{j}^{1}\right\rangle\left\langle\phi_{j}^{1}\right|$ and we assumed that $d_{i j}^{1}$ are the (unknown) coefficients of the density matrix $\rho$ in the basis $\left|\phi_{i}^{1}\right\rangle$. Similarly, for $l=3$, the four states $\left|\phi_{i}^{3}\right\rangle \in\{|0001\rangle,|0010\rangle,|0100\rangle,|1000\rangle\}$ can contribute to $p_{3}$ and $\operatorname{tr}\left(\sum d_{i j}^{3}\left|\phi_{i}^{3}\right\rangle\left\langle\phi_{j}^{3}\right|\right)=p_{3}$. For $l=2$, there are 6 states which might contribute to $p_{2}$, namely $\left|\phi_{i}^{2}\right\rangle \in\{|0011\rangle,|0101\rangle,|0110\rangle,|1001\rangle,|1010\rangle,|1100\rangle\}$ and again $\operatorname{tr}\left(\sum d_{i j}^{3}\left|\phi_{i}^{3}\right\rangle\left\langle\phi_{j}^{3}\right|\right)=p_{3}$. Note that the coefficients $d_{i j}^{l}$ are not specified by the provided data, but the diagonal coefficients $d_{i i}^{l}$ are bound from above by $p_{l}$. We have e.g. that $2 \lambda_{001}=d_{11}^{1}+d_{22}^{3}$. Since we might get contributions to $\lambda_{001}$ from $p_{1}$ and $p_{3}$, we conclude that $0 \leq 2 \lambda_{001} \leq p_{1}+p_{3}$. A similar argument holds e.g. for $2 \lambda_{110}=d_{66}^{2}+d_{11}^{2}$, but in this case we only get contri- butions from $p_{2}$. We thus conclude that $0 \leq 2 \lambda_{110} \leq p_{2}$. The same line of argumentation can be used to obtain bounds for all $\lambda_{k}$. In the experiment, the values of $p_{j}$ were determined to be $p_{1}=p_{2}=p_{3}=0.10 \pm 0.02$.

[19] C. H. Bennett, G. Brassard, S. Popescu, B. Schumacher, J. A. Smolin and W. K. Wootters, Phys. Rev. Lett. 76, 722 (1996); C. H. Bennett, H. J. Bernstein, S. Popescu and B. Schumacher, Phys. Rev. A 53, 2046 (1996).

[20] C. H. Bennett, G. Brassard, C. Crépeau, R. Jozsa, A. Peres, and W. K. Wootters, Phys. Rev. Lett. 70, 1895 (1993). 